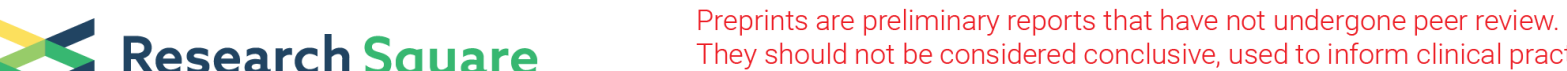 They should not be considered conclusive, used to inform clinical practice, or referenced by the media as validated information.
}

\section{Weak arene/nickel interaction lights on monoarylation of alcohols}

\section{Zhiyan Huang ( $\nabla$ zyhuang@snnu.edu.cn )}

Shaanxi Normal University https://orcid.org/0000-0001-8151-6188

\section{Kun Xie}

Shaanxi Normal University

\section{Ge Meng}

Shaanxi Normal University

Jun He

Sichuan University

\section{Mingsong Shi}

Sichuan University

Junjie Ma

Shaanxi Normal University

\section{Huan Gao}

Shaanxi Normal University

Tingting Qiu

Shaanxi Normal University

\section{Yuxia Zhao}

Shaanxi Normal University

\section{Shuang Yang}

Shaanxi Normal University

\section{Xinyu Wei}

Shaanxi Normal University

Jiaxin Yan

Shaanxi Normal University

\section{Kang Wang}

Shaanxi Normal University

\section{Jiadong Cai}

Shaanxi Normal University

\section{Sheng Li}

Shaanxi Normal University

\section{Dong Xue}

Shaanxi Normal University 


\section{Physical Sciences - Article}

Keywords: nickel catalysis, arylation

Posted Date: August 11th, 2021

DOl: https://doi.org/10.21203/rs.3.rs-771338/v1

License: (c) (i) This work is licensed under a Creative Commons Attribution 4.0 International License. Read Full License 


\section{Abstract}

Despite a growing body of work in nickel catalysis, the potential of organo-photocatalyst serving both as an arene ligand and a sensitizer remains underexplored. Here, we describe such an organo-photocatalyst to promote arylation of alcohols. Mechanistic studies suggest that the formation of sandwich complex via weak arene/nickel interaction and the resulting dexter energy transfer be responsible for this activity. This interaction provides a mechanistically alternative strategy for challenging carbon-oxygen bond assembly, where the elementary steps in transition metal catalysis previously facilitated by intermolecular photoevents replaced by more efficient intramolecular ones. With only $0.05 \mathrm{~mol} \%$ photocatalyst loading and $8 \mathrm{~mol} \%$ nickel bromide, a series of alcohols, diols and triols were (mono)arylated with (hetero)aryl bromides and chlorides. Importantly, many bioactive molecules and active pharmaceutical ingredients containing multiple hydroxyl groups could be efficiently monoarylated, too. This work provides a new approach to access bioactive aryl ethers, but also lights on a highly desirable direction to manipulate the catalytic reactivity of earth abundant nickel.

\section{Main Text}

Visible light involved transition metal (TM) catalysis has expanded rapidly in modern organic synthesis for its utopic TM and substrate activation manners, mild reaction conditions and sustainable properties ${ }^{1-}$ ${ }^{5}$. In the presence of a photocatalyst (PC), the metallaphotocatalysis in current study is realized in cooperation with a transition metal complex through intermolecular photoevents with respect to precious metal $\mathrm{PC}^{6-10}$ or organic dye $\mathrm{e}^{11-13}$, respectively (Fig. $1 \mathrm{~A}-\mathrm{I}$ ). The photocatalyst generally does not directly participate in bond-breaking and bond-forming process, and in most cases ancillary ligands are indispensable to stabilize TM complex during the whole catalytic reaction. While utilizing intramolecular photoevents as a principle, photocatalyst therefore serves both as ancillary ligand to coordinate with TM and a sensitizer to absorb photon energy, is underdevelopment (Fig. 1A-II) ${ }^{14-18}$.

It's well-known that arene could serve as a supporting ligand in $\mathrm{TM}$ catalysis ${ }^{19}$, we envisioned that organo-photocatalyst $(\mathrm{OPC})^{20,21}$, composed with a conjugated polyarene core, may act as an arene-like ligand to interact with transition metals. Under visible light irradiation, this kind of hapto interaction between OPC and nickel could trigger the fundamental steps in catalytic cycle via intramolecular dexter energy transfer (DEnT) ${ }^{22}$, such as oxidative addition (OA) and reductive elimination (RE) (Fig. 1B). This kind of OPC would possess both advantages of sensitizer and ligand. Based on our hypothesis and welldeveloped BDP (boron-dipyrromethene) chemistry ${ }^{23}$, we investigated the possibility of arene/nickel interaction between Et-DABDP $\left(\mathrm{Et}=\right.$ ethyl, $\mathrm{DABDP}=4,4^{\prime}$-diaryl-BDP $)$ and $\mathrm{Ni}(\operatorname{cod})_{2}(\operatorname{cod}=1,5-$ cyclooctadiene). Interestingly, during collection of UV/Vis spectrum, when excess $\mathrm{Ni}(\operatorname{cod})_{2}$ was added into the solution of Et-DABDP, a new absorptive peak appeared obviously around $300 \mathrm{~nm}$ and the undulation of new/original signals was correlated well with the concentration of $\mathrm{Ni}^{0}$, which strongly suggested that a significant association of these two components take place (Fig. 1D). Based on X-ray diffraction analysis, we found that the orientation of two 3,5-di-tert-butylphenyl groups in Et-DABDP is 
unique, one of benzene ring faced towards BDP plane, while another was vertical to BDP plane. Accordingly, through density functional theory (DFT) calculations, two types of arene/nickel complex are obtained, the assemble of Et-DABDP and $\mathrm{Ni}^{0}$ via hapto bonding are calculated to be exergonic by 80.6 and $58.6 \mathrm{kcal} / \mathrm{mol}$, respectively (Fig. 1C, see supplementary materials). The sandwich coordination manner is superior to allyl/Ni interaction by $22 \mathrm{kcal} / \mathrm{mol}$. Inspired by the preliminary findings, here we describe an Et-DABDP/nickel catalyzed (mono)arylation of a variety of alcohols, diols and triols, a wellknown challenging coupling reaction ${ }^{6,24,25}$, with (hetero)aryl bromides and chlorides in the presence of white light, a process that lies at the weak hapto interaction of Et-DABDP core with nickel (Fig. 1E).

We commenced on our study with commercial available Et-BDP as an arene-like ligand to investigate the catalytic potential with 4-bromobenzonitrile 1 and benzyl alcohol $\mathbf{2}$ as coupling partners in the presence of nickel(II) bromide and white LED irradiation. Surprisingly, the coupling reaction could take place in $73 \%$ yield at only 0.05 mol\% loading of Et-BDP after 24 hours (table 1, entry 4). The partial conversion was probably caused by catalyst degradation since 4,4'-difluoro substituted moiety of Et-BDP are known both base $^{26}$ and alcohol ${ }^{27}$ sensitive. We therefore developed several 4,4'-diaryl substituted derivatives to further study the 0 -arylation ${ }^{22}$. As expected, once simply substitution of fluorine with aryl group, the conversion of $\mathbf{1}$ was remarkably improved and the bulkiest Et-DABDP afforded desired $\mathbf{3}$ in $95 \%$ yield (table 1, entries 1-3). The aryl substitution can stabilize the BDP core, but also participate the coordination to nickel (vide supra). It's worth to mention that Ru- and Ir-photocatalyst could catalyze the reaction without addition of ligand ${ }^{6}$, despite moderate conversion (entries 5-7). Control experiments (entries 8-11) suggested that the Et-DABDP/Ni catalyst be vital to form C-O bond (more details see supplementary materials, tables S3-S12).

With the optimal OPC in hand, we firstly examined the type of intramolecular photoevents between EtDABDP and zerovalent nickel. The interaction was further confirmed by Stern-Volmer luminescence quenching experiments recorded by steady-state emission data. Along with the addition of $\mathrm{Ni}(\mathrm{cod})_{2}$, the luminescence intensity of Et-DABDP dramatically decreased, which was in good linear correlation with concentration of $\mathrm{Ni}(\mathrm{cod})_{2}$ (see supplementary materials). The corresponding time-resolved emission spectroscopy (i.e., $I_{0} / \mathrm{I}$, where $\mathrm{I}_{0}$ and $\mathrm{I}$ are the integrated emission intensities of Et-DABDP in the absence and presence of nickel, respectively) afforded a linear equation and almost unchanged fluorescence lifetime (i.e., $t_{0} / t » 1$, where $t_{0}$ and $t$ are the fluorescence lifetime of Et-DABDP in the absence and presence of nickel, respectively) (Fig. 2A). The latter data strongly suggested that static quenching probably proceed between Et-DABDP and zerovalent nickel under visible light irradiation, which was in good support of dexter energy transfer (DEnT) mechanism during activation of Ni species $7,22,28$.

We next examined OA efficiency and possible OA pathway of our Et-DABDP/Ni catalyst ${ }^{29-31}$. Using electron-neutral bromide $\mathbf{4}$ as electrophile, in-situ generated "OA active species" consumed $\mathbf{4}$ in the presence of $\mathrm{Ni}(\mathrm{cod})_{2}$ and lead to the formation of self-coupling product 5 via disproportionation of nickel(II) intermediate. In sharp contrast, poor conversion was observed under dark condition, which highlighted the OA efficiency of induced by Et-DABDP (Fig. 2B). The main concern about the OA pathway 
between excited nickel and aryl electrophile is whether aryl radical involved or not. To understand more details, we examined the coupling reaction of cis-diol 7 with 2-(allyloxy)iodobenzene 6, a radical probe that was frequently used as evidence to support or exclude a radical mechanism ${ }^{14}$. As aryl radical 10 is known to cyclize very fast to produce alkyl radical $11\left[k_{o b s}=9.6 \times 10^{9} \mathrm{~s}^{-1} \text { in DMSO }\right]^{32}$, the identification of cyclized products 12 and 13 would be a valid indicator to support radical mechanism, whereas sole formation of uncyclized $\mathbf{8}$ would rule out the radical route. Interestedly, in our case we observed exclusive production of uncyclized coupling products ( 8 and deprotected 9 in $48 \%$ and $15 \%$ yields, respectively), along with $10 \%$ remaining starting material (SM), some deprotection and deiodination SM (Fig. 2C). Furthermore, the combination of equal mole $\mathrm{NiBr}_{2}$ and $\mathrm{Ni}(\mathrm{cod})_{2}$ which is assumed to in-situ generate nickel(I) species via comproportionation ${ }^{33}$, didn't improve catalytic activity but gave lower yield compared to $\mathrm{NiBr}_{2}$ alone as metal source. And we have not observed the electron paramagnetic resonance (EPR) signal of paramagnetic nickel(I) species ${ }^{25}$. Hence the results from a series of experiments strongly suggested that oxidative addition between in-situ generated $\left[\mathrm{Ni}^{0}\right]^{*}$ and aryl electrophile likely proceed through two-electron pathway to form Et-DABDP/(ArNillBr) oxidative addition adduct. Furthermore, in the product formation step, high valent Et-DABDP/[ArNilll (OR)] may not formed since base DCyMA hardly quenched the luminescence of Et-DABDP 6 (see supplementary materials).

Based on our observations, a proposed catalytic cycle was outlined in Fig. 2D. Et-DABDP firstly acted as an arene ligand to coordinate with zerovalent nickel generated by reduction of $\mathrm{NiBr}_{2}$ in the presence of DCyMA. Upon irradiation of the complex with white LED, the excited OA active nickel species was produced via intramolecular DEnT from excited [Et-DABDP]* to [ $\left.\mathrm{Ni}^{0}\right]$, which in turn afforded excited [ $\left.\mathrm{Ni}^{0}\right]$ * and followed by the formation of OA adduct with aryl halide. With the help of mixed base DCyMA and $\mathrm{Na}_{2} \mathrm{CO}_{3}$, the RE precursor was generated via transmetalation. With respect of product forming step, the intramolecular DEnT in Et-DABDP/[ArNi"(OR)] complex ${ }^{7,34}$ and/or arene-like ligand property of Et-DABDP both should again play crucial roles in facilitating $\mathrm{C}-\mathrm{O}$ bond formation.

With the proof of our concept, we next examined the scope of this Et-DABDP/nickel-catalyzed arylation of alcohol with $0.05 \mathrm{~mol} \% \mathrm{Et}-\mathrm{DABDP}$ and $8 \mathrm{~mol} \% \mathrm{NiBr}_{2}$ loading, respectively. As shown in Fig. 3, we were pleased to find that a wide array of alcohols and aryl electrophiles can be efficiently coupled under the optimal conditions (60-98\% yields). Substituted benzyl-, (fluorinated)alkyl-, allyl-, and amino alcohols are coupled smoothly (entries 14-22, 24, 33, 43). Primary and secondary alcohols bearing amide, ester, ether, ketone, ketal and cyclopropyl functional groups are all tolerated well (entries 23-33). Most importantly, our Et-DABDP/Ni catalyst could selectively give various mono-arylation products in excellent yields with only once fold excess of diols under much milder conditions $\left(40^{\circ} \mathrm{C}\right)$ without addition of inorganic base $\mathrm{Na}_{2} \mathrm{CO}_{3}$. A variety of diols (entries 35-47), including 1,2-, 1,3-1,4-, 1,5-diols and cis/trans cyclic diols are all suitable coupling partners. Methyl $(S)$-lactate containing an enolizable chiral center ${ }^{35-37}$ was suitable coupling partner under our mild conditions (entry $32,87 \%$ yield and $98 \%$ ee), which drove as to expand to other common chiral alcohols (entries 34, 43, 46 and 47). Notably, without external ligand added, challenging electron-rich aryl electrophile ${ }^{6}$ (entries 55 and 61 ) are suitable coupling partner in our new 
protocol despite a little bit lower yield in comparison with electron-neutral and -poor ones. Heteroaryl partners based on quinoline and pyridine skeletons could couple smoothly (entries 57-59). In particular, electron-deficient aryl chlorides are suitable partners, too.

To further demonstrate synthetic diversification of Et-DABDP/Ni-catalyzed C-O cross couplings, a series of important intermediates of bioactive molecules and drugs were prepared and active pharmaceutical ingredients (APIs) or their derivatives were directly arylated using our novel catalyst ${ }^{38}$. As illustrated in Fig. 4. Aryl ethers $\mathbf{7 0}$ and $\mathbf{7 2}$, key intermediate of pioglitazone for managing type II diabetes and pimavanserin for treating hallucinations and psychosis, were obtained smoothly with $90 \%$ and $70 \%$ yields starting with aldehyde $\mathbf{6 8}$, respectively. Monoarylation of glucoside derivative was smoothly proceeded and the desired products $\mathbf{7 3}$ were obtained in $93 \%$ total yields. We next applied our catalyst to directly modify APIs. Encouragingly, a variety of complicated molecules were coupled smoothly, which afforded corresponding arylated derivatives in excellent yields. Ester of rosuvastatin and atorvastatin, two blockbuster drugs derivatives, could be arylated efficiently, which provide separable mono-arylated products $\mathbf{7 5}$ and $\mathbf{7 6}$ in $\mathbf{9 0 \%}$ and $95 \%$ total yields, respectively. Notably, APIs fingolimod, methylprednisolone and cortisol were all monoarylated, which smoothly generated desired derivatives in 91-95\% yields (entries 77-79).

In conclusion, a weak arene/nickel interaction was disclosed between bifunctional organo-photocatalyst Et-DABDP and zerovalent nickel, and utilized in challenging carbon-oxygen bond formation via dexter energy transfer (DEnT). As a mechanistically alternative tactic to facilitate elementary steps of transition metal catalysis, with only $0.05 \mathrm{~mol} \%$ photocatalyst loading and $8 \mathrm{~mol} \% \mathrm{NiBr}_{2}$, Et-DABDP/Ni system achieved both oxidative addition and reductive elimination during the process of monoarylation of alcohols. This work provides a new approach to access bioactive aryl ethers, but also lights on a highly desirable direction to manipulate the catalytic reactivity of nickel. The composition of visible light, OPC and non-precious metal catalyst represents an important current theme in sustainable chemical synthesis. We anticipate that excitation of nickel via intramolecular DEnT is likely to be broadly general and the exploration of an ideal OPC will lead to diverse applications in many types of cross coupling reactions.

\section{Online Content}

Any methods, additional references, Nature Research reporting summaries, source data, extended data, supplementary information, acknowledgements, peer review information; details of author contributions and competing interests; and statements of data and code availability are available at https://doi.org/xxxxx.

\section{Declarations}

\section{Data availability}


The data that support the findings of this study are available from the corresponding author upon reasonable request.

Acknowledgements Z. H. thanks Prof. Zhangjie Shi (Fudan University) for helpful discussion and instruction in mechanism investigation, Prof. Hongzhu Ma (SNNU) for supporting UV/Vis spectra instrument and Dr. Huaming Sun (SNNU) for the X-ray diffraction analyses. We also thank the National Supercomputing Center of Guangzhou in supporting DFT calculation. Funding: This research is supported by the National Natural Science Foundation of China (No. 21872090), and the Natural Science Foundation of Shaanxi Province (No. 2015JM2064 and 2019JM-134), the Fundamental Research Funds for the Central Universities (GK202003033), and Funded Projects for the Academic Leaders and Academic Backbones, SNNU (16QNGG009) for financial support.

Author contributions K. Xie, G. Meng, J. He and S. M. Shi were contributed equally for this work. K. Xie and G. Meng conducted the synthesis of photocatalyst, mechanism investigation, product separation and characterization. J. He and S. M. Shi designed and executed the calculations. J. J. Ma, H. Gao, T. T. Qiu, Y. X. Zhao, S. Yang, X. Y. Wei, J. X. Yan, K. Wang, J. D. Cai and S. Li assisted partial experiments. D. Xue helped to prepare manuscript. Z.Y. Huang was responsible for raising initial conception of the project and preparing this manuscript.

Competing interests The authors declare no competing interests.

\section{Additional information}

Supplementary information The online version contains supplementary material available at https://doi.org/xxx.

Correspondence and requests for materials should be addressed to Z.Y. Huang.

Reprints and permissions information is available at http://www.nature.com/reprints.

\section{References}

1. Prier, C. K., Rankic, D. A. \& MacMillan, D. W. C. Visible Light Photoredox Catalysis with Transition Metal Complexes: Applications in Organic Synthesis. Chem. Rev. 113, 5322-5363 (2013).

2. Schultz, D. M. \& Yoon, T. P. Solar Synthesis: Prospects in Visible Light Photocatalysis. Science 343, 1239176 (2014).

3. Romero, N. A. \& Nicewicz, D. A. Organic Photoredox Catalysis. Chem. Rev. 116, 10075-10166 (2016).

4. Twilton, J. et al. The merger of transition metal and photocatalysis. Nature Reviews Chemistry 1, 0052 (2017). 
5. Stephenson, C. R. J., Yoon, T. P., MacMillan, D. W. C. Eds., Visible Light Photocatalysis in Organic Chemistry (Wiley, 2018).

6. Terrett, J. A., Cuthbertson, J. D., Shurtleff, V. W. \& MacMillan, D. W. C. Switching on elusive organometallic mechanisms with photoredox catalysis. Nature 524, 330-334 (2015).

7. Welin, E. R., Le, C., Arias-Rotondo, D. M., McCusker, J. K. \& MacMillan, D. W. C. Photosensitized, energy transfer-mediated organometallic catalysis through electronically excited nickel(II). Science 355, 380-385 (2017).

8. Le, C., Liang, Y., Evans, R. W., Li, X. \& MacMillan, D. W. C. Selective sp3 C-H alkylation via polaritymatch-based cross-coupling. Nature 547, 79-83 (2017).

9. Tellis, J. C., Primer, D. N. \& Molander, G. A. Single-electron transmetalation in organoboron crosscoupling by photoredox/nickel dual catalysis. Science $345,433-436$ (2014).

10. Johnston, C. P., Smith, R. T., Allmendinger, S. \& MacMillan, D. W. C. Metallaphotoredox-catalysed sp3sp3 cross-coupling of carboxylic acids with alkyl halides. Nature 536, 322-325 (2016).

11. Yang, L. et al. Synthesis of Phenols: Organophotoredox/Nickel Dual Catalytic Hydroxylation of Aryl Halides with Water. Angew. Chem. Int. Ed. 57, 1968-1972 (2018).

12. Lu, J. et al. Donor-Acceptor Fluorophores for Energy-Transfer-Mediated Photocatalysis. J. Am. Chem. Soc. $140,13719-13725$ (2018).

13. Luo, J. \& Zhang, J. Donor-Acceptor Fluorophores for Visible-Light-Promoted Organic Synthesis: Photoredox/Ni Dual Catalytic C(sp3)-C(sp2) Cross-Coupling. ACS Catalysis 6, 873-877 (2016).

14. Creutz, S. E., Lotito, K. J., Fu, G. C. \& Peters, J. C. Photoinduced Ullmann C-N Coupling: Demonstrating the Viability of a Radical Pathway. Science 338, 647-651 (2012).

15. Kainz, Q. M. et al. Asymmetric copper-catalyzed C-N cross-couplings induced by visible light. Science 351, 681-684 (2016).

16. Torres, G. M., Liu, Y. \& Arndtsen, B. A. A dual light-driven palladium catalyst: Breaking the barriers in carbonylation reactions. Science 368, 318-323 (2020).

17. Xia, Z. et al. Photosensitized oxidative addition to gold(i) enables alkynylative cyclization of oalkylnylphenols with iodoalkynes. Nature Chemistry 11, 797-805 (2019).

18. Cheng, W.-M. \& Shang, R. Transition Metal-Catalyzed Organic Reactions under Visible Light: Recent Developments and Future Perspectives. ACS Catalysis 10, 9170-9196 (2020).

19. Walton, J. W. \& Wilkinson, L. A. in Organometallic Chemistry: $\pi$-Coordinated arene metal complexes and catalysis Vol. 42, 125-171 (The Royal Society of Chemistry, 2019). 
20. MacKenzie, I. A. et al. Discovery and characterization of an acridine radical photoreductant. Nature 580, 76-80 (2020).

21. Vega-Peñaloza, A., Mateos, J., Companyó, X., Escudero-Casao, M. \& Dell'Amico, L. A Rational Approach to Organo-Photocatalysis: Novel Designs and Structure-Property Relationships. Angew. Chem. Int. Ed. 60, 1082-1097 (2021).

22. Arias-Rotondo, D. M. \& McCusker, J. K. The photophysics of photoredox catalysis: a roadmap for catalyst design. Chem. Soc. Rev. 45, 5803-5820 (2016).

23. Loudet, A. \& Burgess, K. BODIPY Dyes and Their Derivatives: Syntheses and Spectroscopic Properties. Chem. Rev. 107, 4891-4932 (2007).

24. Han, R. \& Hillhouse, G. L. Carbon-Oxygen Reductive-Elimination from Nickel(II) Oxametallacycles and Factors That Control Formation of Ether, Aldehyde, Alcohol, or Ester Products. J. Am. Chem. Soc. 119, 8135-8136 (1997).

25. Yang, L., Simionescu, R., Lough, A. \& Yan, H. Some observations relating to the stability of the BODIPY fluorophore under acidic and basic conditions. Dyes and Pigments 91, 264-267 (2011).

26. Wang, M., Vicente, M. G. H., Mason, D. \& Bobadova-Parvanova, P. Stability of a Series of BODIPYs in Acidic Conditions: An Experimental and Computational Study into the Role of the Substituents at Boron. ACS Omega 3, 5502-5510 (2018).

27. Ma, P., Wang, S. \& Chen, H. Reactivity of Transition-Metal Complexes in Excited States: C-O Bond Coupling Reductive Elimination of a Ni(II) Complex Is Elicited by the Metal-to-Ligand Charge Transfer State. ACS Catalysis 10, 1-6 (2020).

28. Tsou, T. T. \& Kochi, J. K. Mechanism of oxidative addition. Reaction of nickel(0) complexes with aromatic halides. J. Am. Chem. Soc. 101, 6319-6332 (1979).

29. Diccianni, J. B. \& Diao, T. Mechanisms of Nickel-Catalyzed Cross-Coupling Reactions. Trends in Chemistry 1, 830-844 (2019).

30. Malik, J. A., Madani, A., Pieber, B. \& Seeberger, P. H. Evidence for Photocatalyst Involvement in Oxidative Additions of Nickel-Catalyzed Carboxylate O-Arylations. J. Am. Chem. Soc. 142, 11042-11049 (2020).

31. Giri, R. \& Hartwig, J. F. Cu(I)-Amido Complexes in the Ullmann Reaction: Reactions of $\mathrm{Cu}(\mathrm{I})$-Amido Complexes with lodoarenes with and without Autocatalysis by Cul. J. Am. Chem. Soc. 132, 15860-15863 (2010).

32. Mohadjer Beromi, M. et al. Mechanistic Study of an Improved Ni Precatalyst for Suzuki-Miyaura Reactions of Aryl Sulfamates: Understanding the Role of Ni(I) Species. J. Am. Chem. Soc. 139, 922-936 
(2017).

33. Yang, L. et al. Light-Promoted Nickel Catalysis: Etherification of Aryl Electrophiles with Alcohols Catalyzed by a Nill-Aryl Complex. Angew. Chem. Int. Ed. 59, 12714-12719 (2020).

34. Tian, L., Till, N. A., Kudisch, B., MacMillan, D. W. C. \& Scholes, G. D. Transient Absorption Spectroscopy Offers Mechanistic Insights for an Iridium/Nickel-Catalyzed C-O Coupling. J. Am. Chem. Soc. 142, 45554559 (2020).

35. Huang, Z. Y., Liu, Z. \& Zhou, J. R. An Enantioselective, Intermolecular alpha-Arylation of Ester Enolates To Form Tertiary Stereocenters. J. Am. Chem. Soc. 133, 15882-15885 (2011).

36. Huang, Z. et al. Weak Arene C-H center dot center dot center dot O Hydrogen Bonding in PalladiumCatalyzed Arylation and Vinylation of Lactones. Angew. Chem.Int. Ed. 52, 5807-5812 (2013).

37. Huang, Z. et al. Arene $\mathrm{CH}-\mathrm{O}$ Hydrogen Bonding: A Stereocontrolling Tool in Palladium-Catalyzed Arylation and Vinylation of Ketones. Angew. Chem.Int. Ed. 52, 4906-4911 (2013).

38. Uehling, M. R., King, R. P., Krska, S. W., Cernak, T. \& Buchwald, S. L. Pharmaceutical diversification via palladium oxidative addition complexes. Science 363, 405-4083 (2019).

\section{Table 1}

Due to technical limitations, Table 1 is only available in the supplementary files section.

\section{Figures}


A

$1 \mathrm{M}^{\mathrm{N}}-\mathrm{IM]}^{\mathrm{B}}$

With precious metal PC

Intermolecular photoevents

II

$\mathrm{D}-\mathrm{A}-[\mathrm{M}]$

Weak

arene/metal interaction

This work: Intramolecular photoevents

c

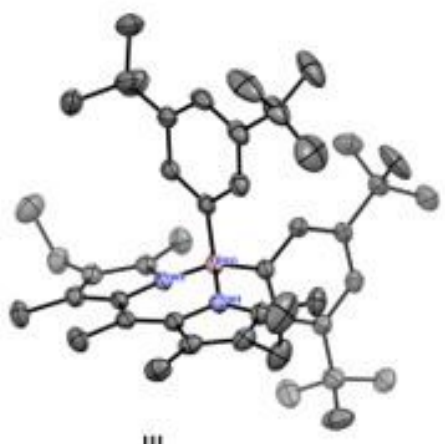

III

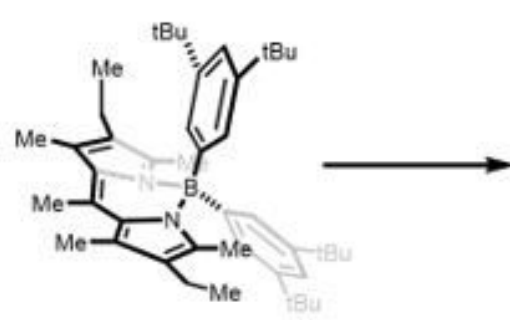

( $0 \mathrm{kcal} / \mathrm{mol}$ )

Et-DABDP + $\left[\mathrm{Ni}^{0}\right]$
B

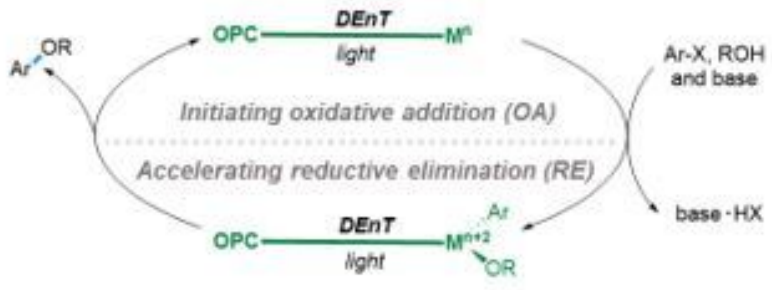

Dexter energy transfer (DEnT)

D

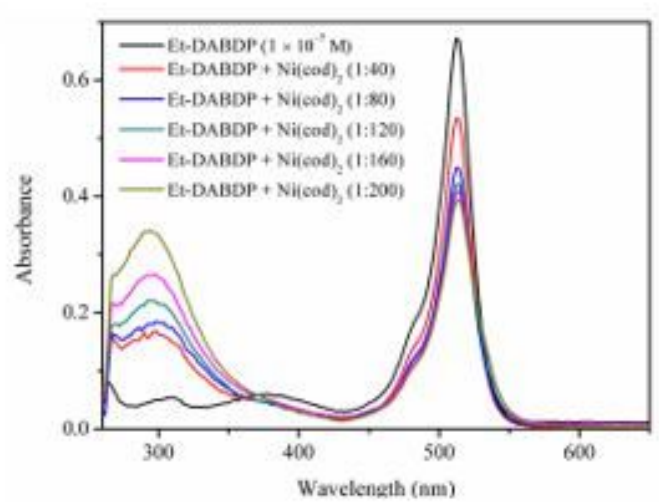

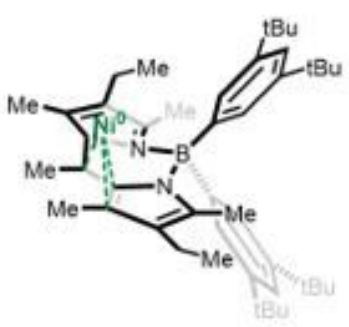

( $-80.6 \mathrm{kcal} / \mathrm{mol})$

$(-58.6 \mathrm{kcal} / \mathrm{mol})$ Et-DABDP/Ni-A3

As an arene ligand and organo-photocatalyst, Et-DABDP weakly coordinates with nickel and efficiently facilitates $O A$ and RE steps via intramolecular photoevents.

E

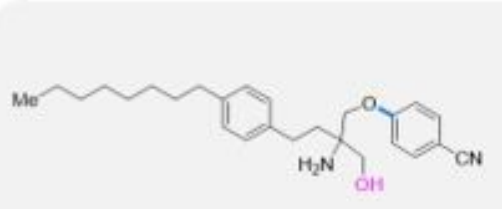

Fram fropannod

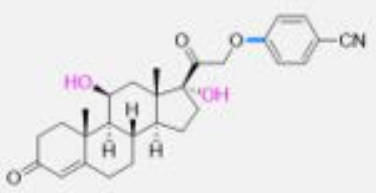

From cortsol

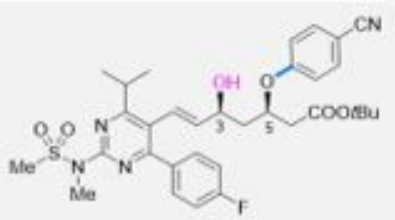

from rosivastaty

- Bifunctional organophotocatalyst

- Dual Photoevents

- Broadly arylation of alcohols

- Feedstock reagents

\section{Figure 1}

Weak Et-DABDP/nickel interaction lights on arylation of alcohols. A, Light-induced intermolecular photoevents and intramolecular photoevents, M, metal; D, donor; A, acceptor. B, Intramolecular dexter energy transfer (DEnT) in arylation of alcohol. C, X-ray structure of Et-DABDP (thermal ellipsoids are drawn at $50 \%$ probability and $\mathrm{H}$ atoms are omitted for clarity), calculated geometry and Gibbs energy 
change of Et-DABDP/[NiO]m interaction. D, UV-Vis spectrum outcome of Et-DABDP/Ni(cod)2, E, EtDABDP/Ni catalyzed efficient arylation of active pharmaceutical ingredients (APIs).

A

luminescence quenching study with zerovalent nickel

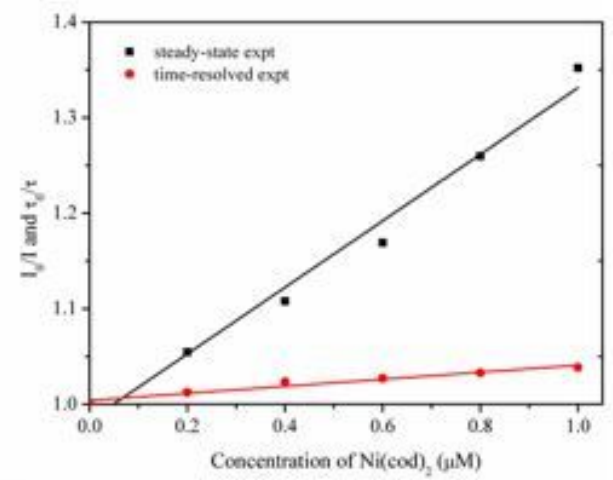

B
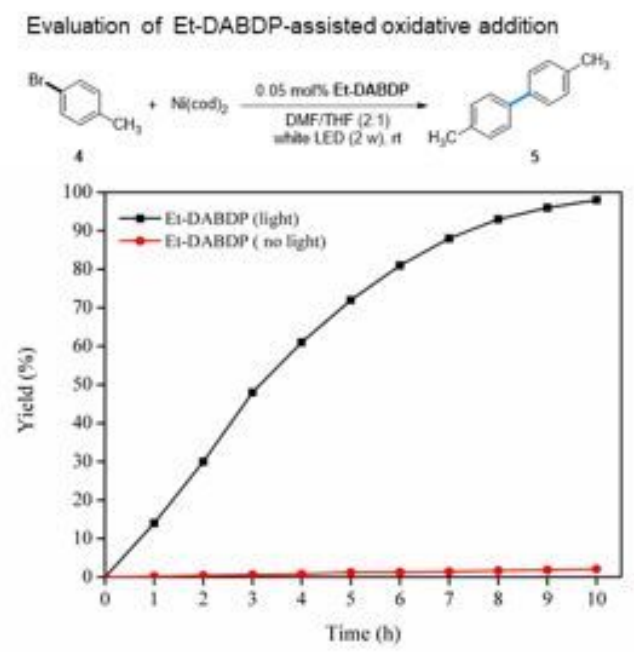

C

Experiments observations supporting concerted oxidative addition pathway
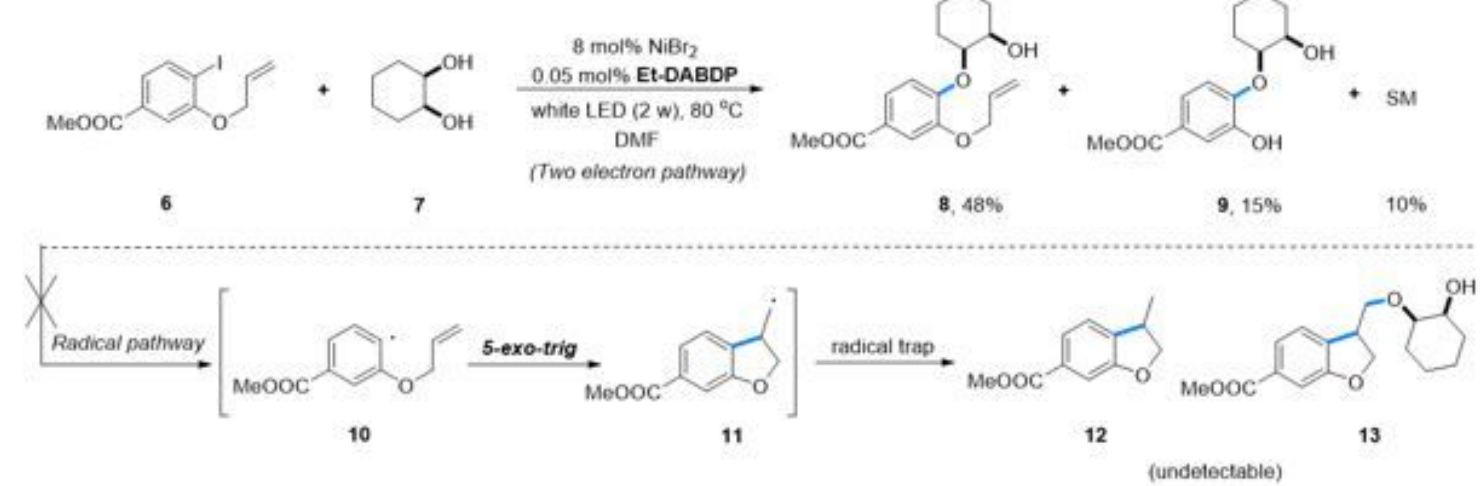

D A proposed catalytic cycle of Et-DABDP/Ni catalyzed arylation of alcohol
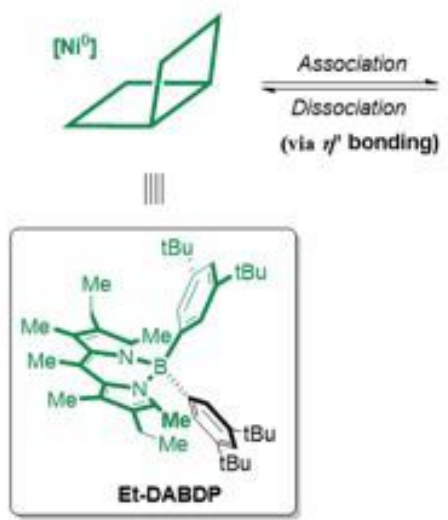
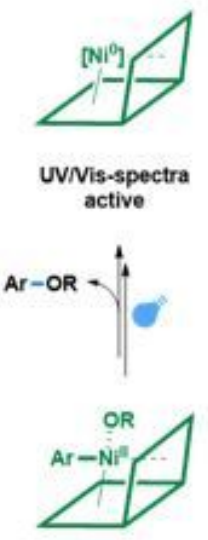

Reductive elimination precursor
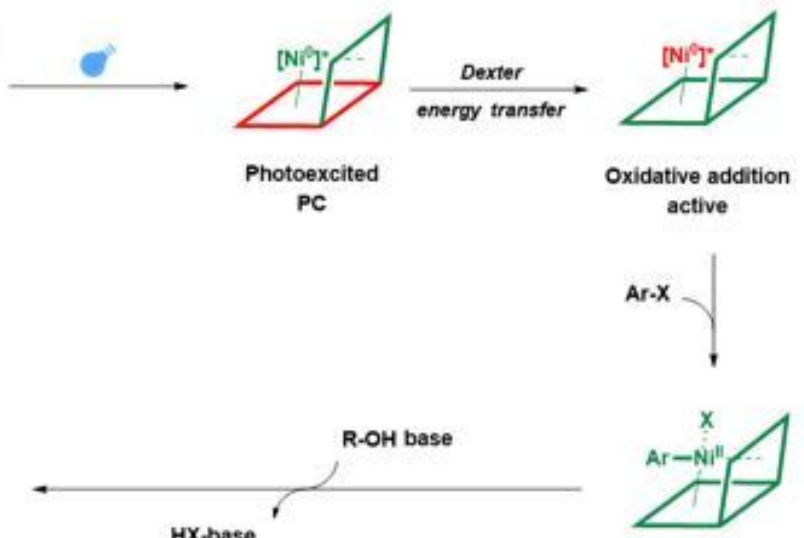

HX-base

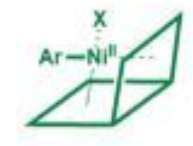

Oxidative addition adduct

\section{Figure 2}

Mechanistic studies and a proposed catalytic cycle. 

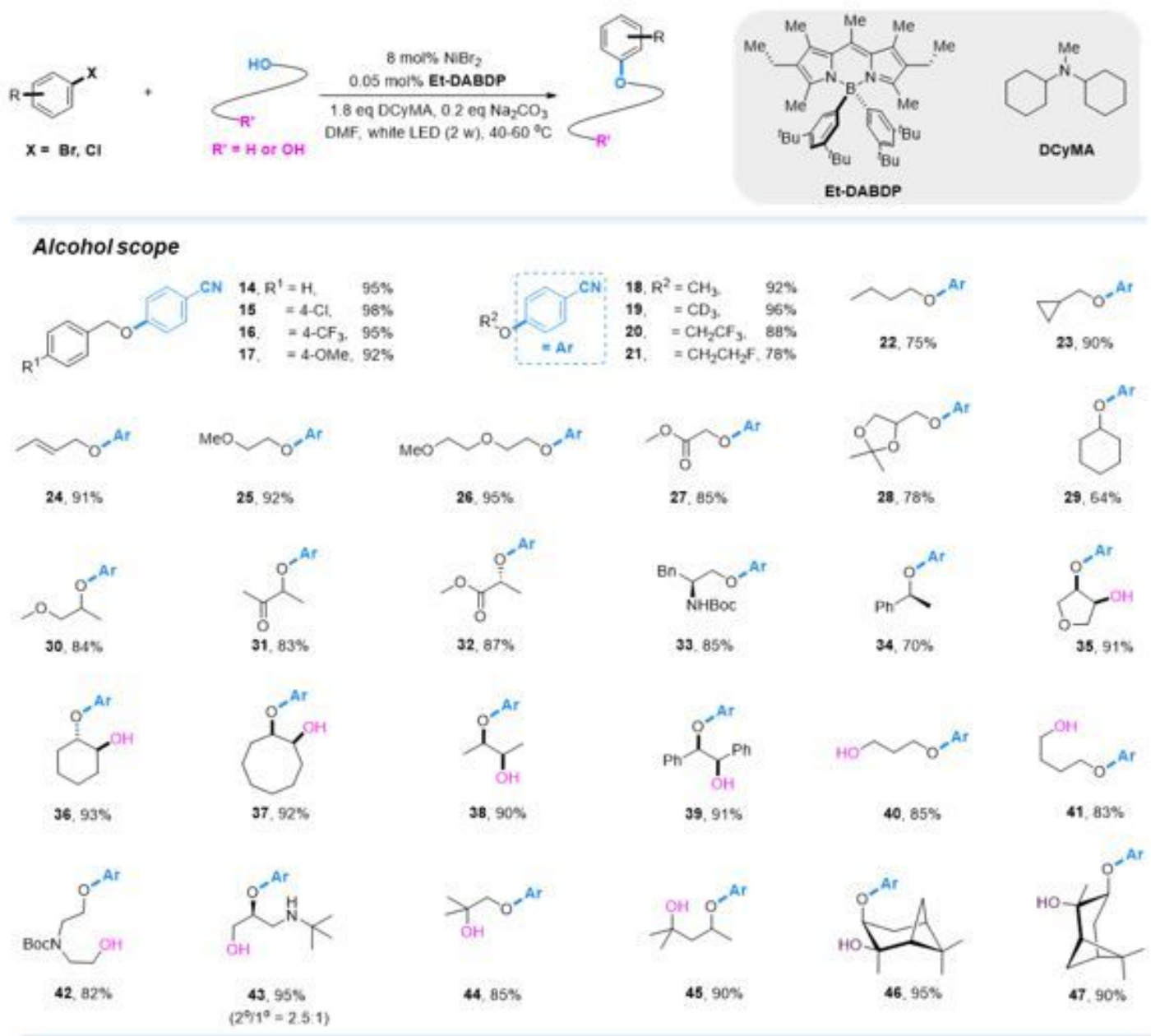

\section{Aryl electrophile scope}

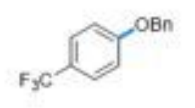

48, 815

54. $78 \%$

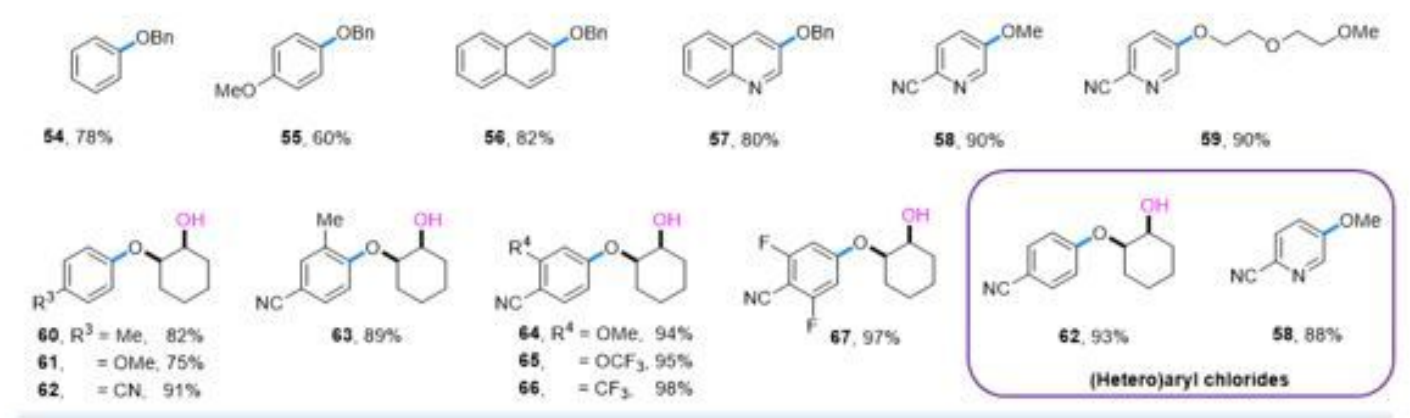

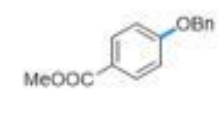

$49,85 \%$

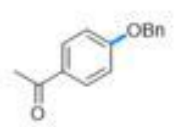

so, $80 \%$

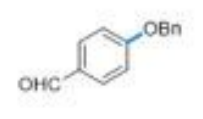

$51,95 \%$

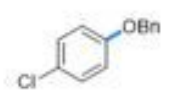

52, $82 \%$

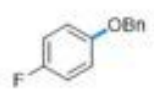

53, $81 \%$

\section{Figure 3}

Scopes of Et-DABDP/Ni in (mono)arylation of alcohols. Yields were determined by isolation after chromatographic purification. Diols as coupling partners, $\mathrm{Na} 2 \mathrm{CO} 3$ was not necessary. CF3, trifluoromethyl group; $\mathrm{CD} 3$, trideuteromethyl group; OMe, methoxy group; Me, methyl group; Bn, benzyl group; Ph, phenyl group; Boc, tert-butoxy carbonyl group; CN, nitrile group. 


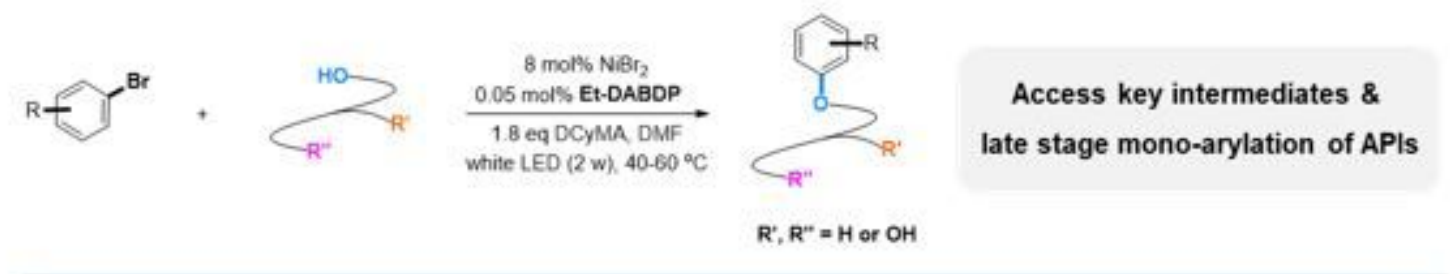

\section{Preparing key intermediates of APIs}
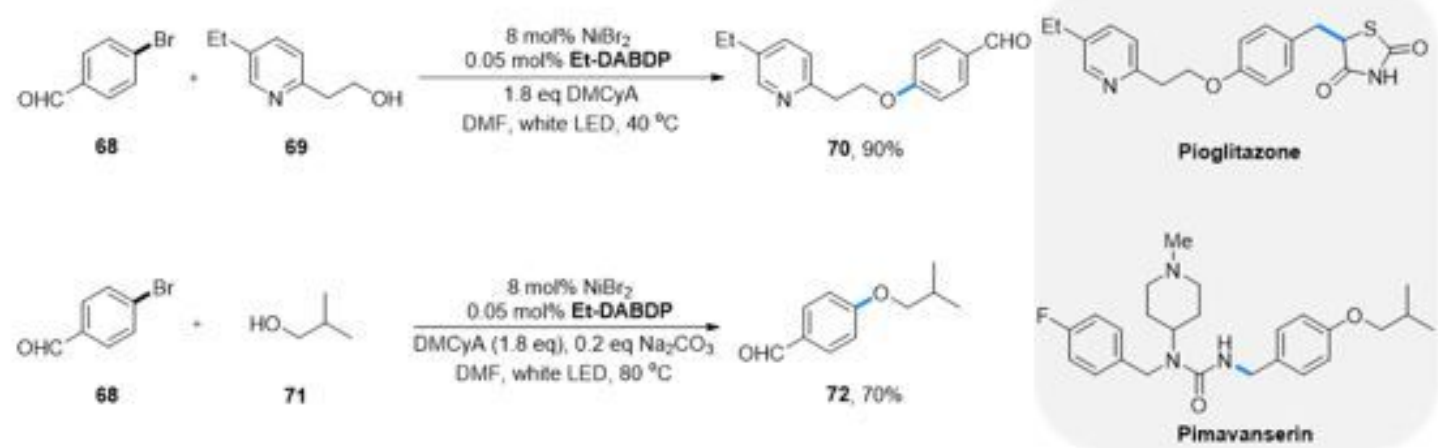

\section{Late stage functionalization of bioactive molecules \& APls}
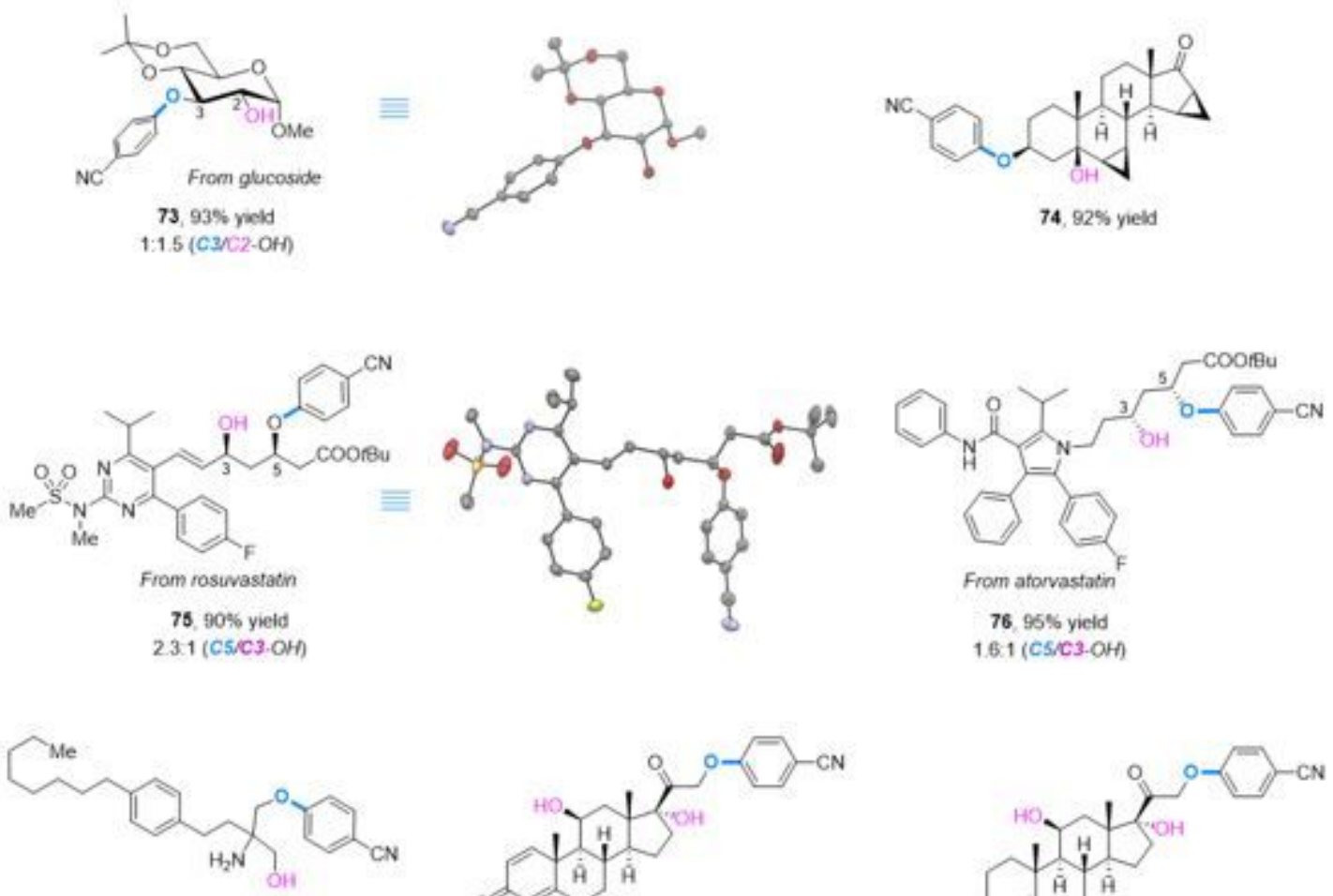

From fingolimod

$77,91 \%$ yield

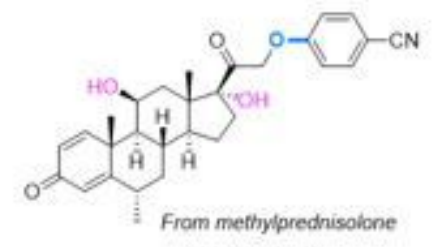

78, $92 \%$ yield

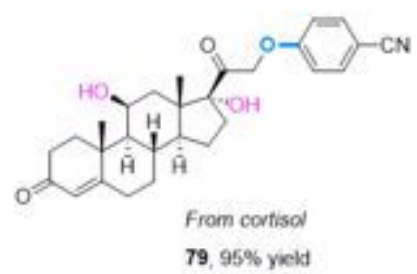

\section{Figure 4}

Application of Et-DABDP/Ni in drug formal synthesis and modification. Yields were determined by isolation after chromatographic purification. APIs, active pharmaceutical ingredients; Et, ethyl group; tBu, tert-butyl group.

\section{Supplementary Files}


This is a list of supplementary files associated with this preprint. Click to download.

- CCDC2098221.cif

- CCDC2098343.cif

- CCDC2098229.cif

- CCDC2098228.cif

- Supplementaryinformationformanuscript.docx 